\title{
PROCESS CAPABILITY ANALYSIS IN PHARMACEUTICAL PRODUCTION
}

\author{
Mohammed Raihan Chowdhury
}

\section{Introduction}

A pharmaceutical company produces products such as tablets, capsules as per customer requirements. Customer requirements are the significant features that the customers expect to find in a product. Development scientists translate those requirements into 'Critical-toQuality' (CTQ) characteristics of the products that they are about to produce. As example, hardness, thickness, uniformity of weight, assay, dissolution etc are CTQ characteristics of tablets. When these CTQ characteristics are assessed and quality targets are determined, development scientists specify upper and lower limit within which variables of CTQ characteristics must fall. To evaluate production process performance, the CTQ characteristics are monitored when production in progress. Control chart is one of the tools which used to monitor the characteristics. We can see whether the data of CTQ characteristics are within limit or not from control chart. Even if all the monitored data are within set limit, there is a possibility to produce defective product. The tool by which we can say the process is capable to produce product complying customers' requirements is process capability index Cpk.

\section{Control Chart and Process Capability}

While the production is in progress, the performance of the production process is monitored to detect and prevent possible variations. The tool frequently used to monitor a process performance while the production is in progress is the control chart. It helps detect assignable causes of variations and facilitate corrective actions. But a control chart is not the correct tool to determine if the customers' requirements are met because it is only used to monitor the performance of production processes in progress, and an in-control process does not necessarily mean that all the products meet the customers' (or set limit) requirements. In other words, a process can be contained within the upper and lower control limits and still generate products that are outside the specified limits. Here, two terms should be clear. Customers' requirement or set limit is the standard specified limit which is 'voice of the customer' and control limit is n sigma limit ( $\mathrm{n}$ may be 3 ) which is generated from monitored data \& which is 'voice of the process'.

Suppose that we are monitoring a compression process of a product $\mathrm{X}$ and that the thickness of the product is critical to quality (CTQ). Control chart of Figure 1 plot the measurements that were obtained while monitoring the compression process. Remember that control charts are constructed by taking samples from the production line at preset intervals and plotting the means of the samples on a chart.

\footnotetext{
Correspondence to author:

E-mail: raihan c36@yahoo.com

Cell: +8801717061037
} 


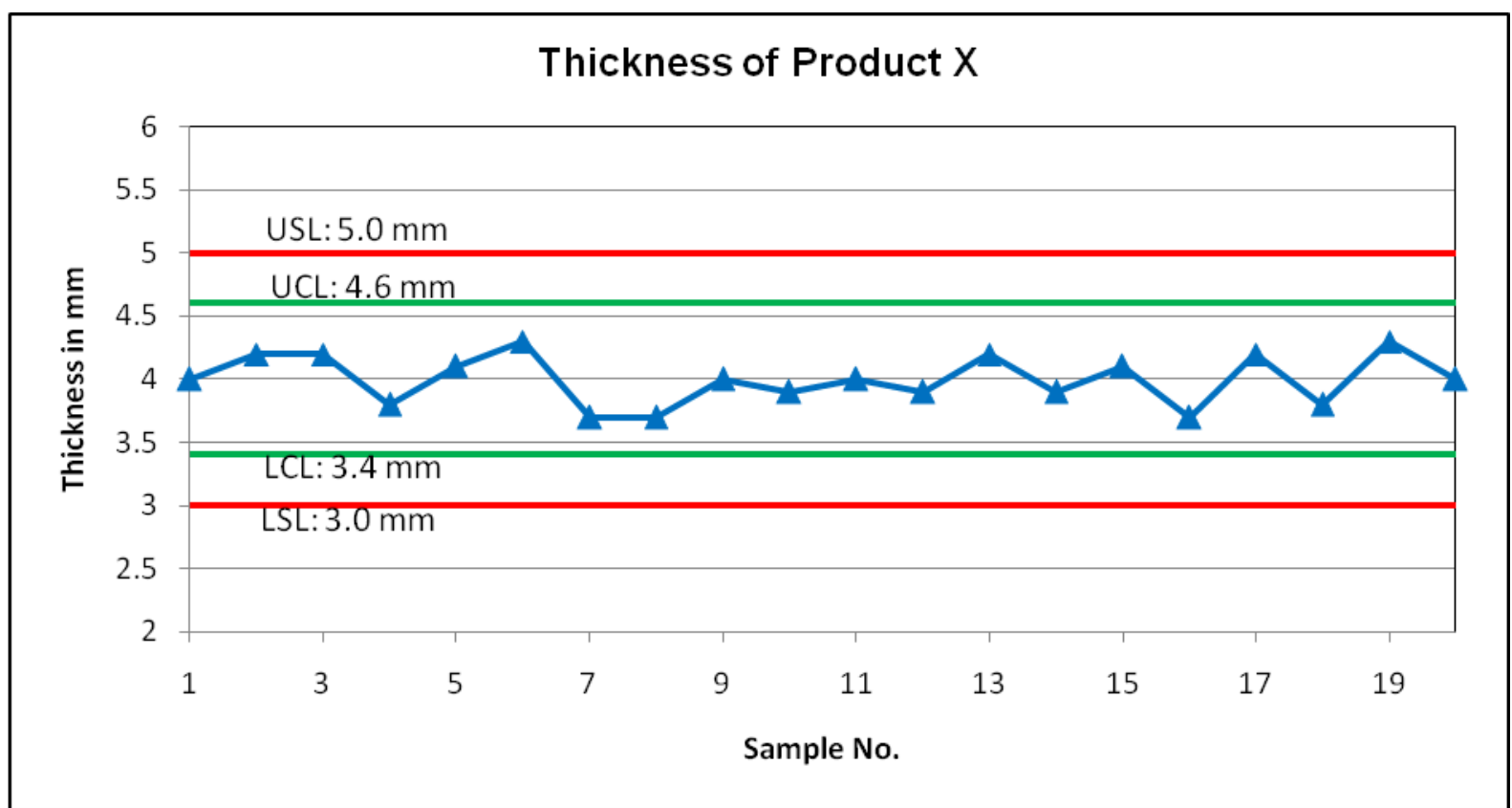

Figure-1: Thickness of product $X$ (capable process)

From the observation of the compression process that generated the graph of Figure-1, we can conclude that the process is acceptably stable and that the variations are within the control limits that is within UCL \& LCL. Yet we cannot conclude that all the output meets the customers' expectations. Because control limit ( $n$ sigma limit) might be within standard specified limit or outside of standard specified limit. The output in this case is within the UCL (4.6) and the LCL (3.4). If the standard specified limits were 4.0 for the lower specified limit (LSL) and 6.0 for the upper specified limit (USL), some of the parts produced by the machine would have met the customers' expectations and some not. In other words, a portion would have been considered as defective. So a stable and in-control production process does not necessarily mean that all the output meets customers' requirements or standard specified limit. To understand the process capability, it is required to relate standard specified limit (voice of the customer) with control limit (voice of the process).

The control charts do not relate the process performance to the customers' requirements because there is not any statistical or mathematical relationship between the standard specified limits and the process control limits. The process capability analysis is the bridge between the two; it compares the variability of an in-control and stable production process with its standard specified limit and capability indices are generated to measure the level of the process performance as it relates to the customers' requirements. A process is said to be capable if the process mean is centered to the specified target and the range of the specified limits is wider than the one of the actual production process variations (Control Limits), as in the graph in Figure-1.

If the spread of the natural variations (control limits) is larger than the one of the standard specified limits, as in the example of Figure-2, the process is considered incapable because some of the parts produced are outside the standards specified limit. Though control chart of Figure-2 is showing that all tested data are within standard specified limit, but there is possibility to produce product with defective thickness since control limit is wider than 
standard specified limit. Data plotted in chart are mean value; individual values possess some variation from mean value. So, individual value might be out of standard specified limit in case of Figure-2.

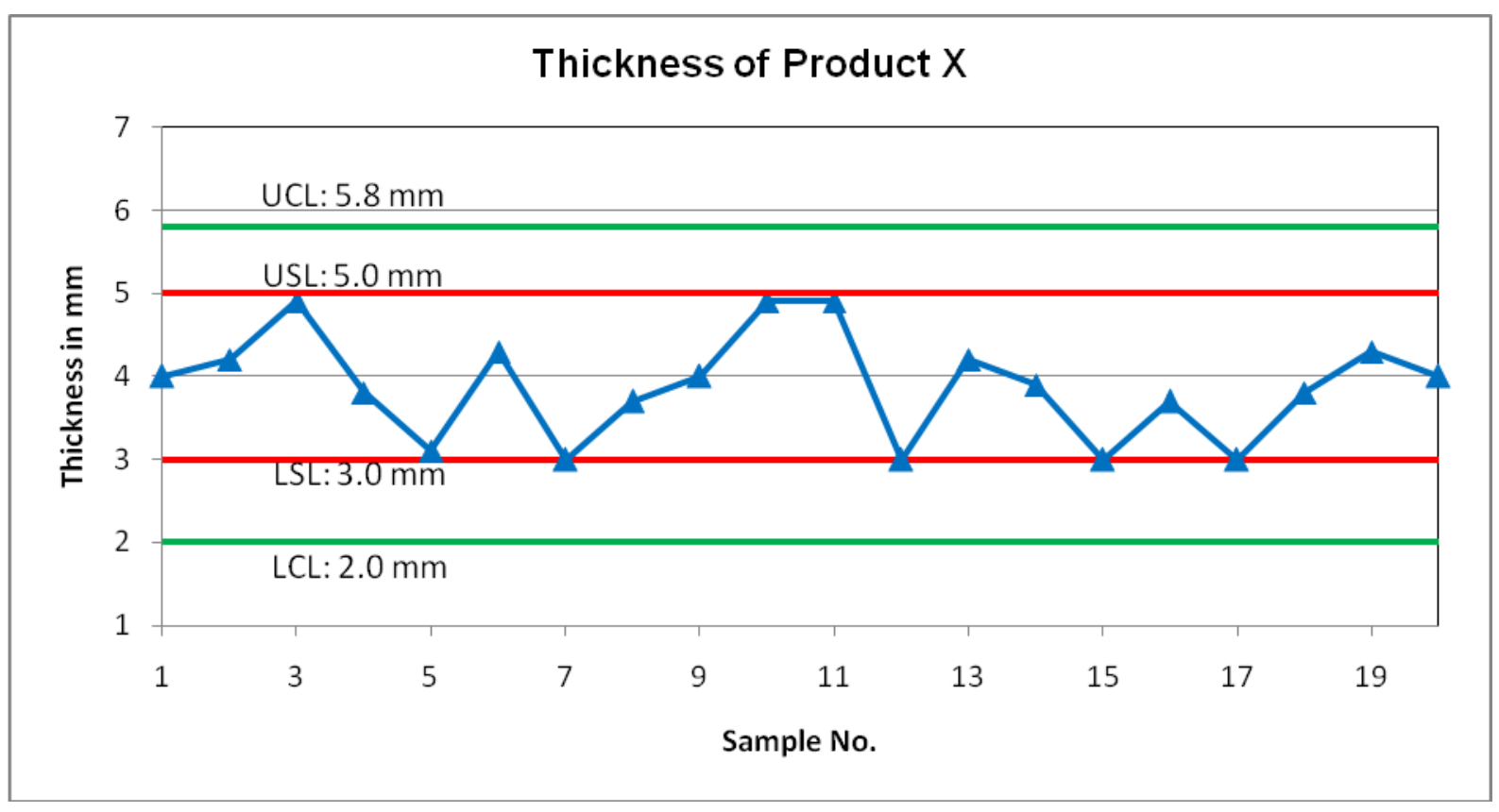

Figure-2: Thickness of Product X (incapable process)

\section{Calculation of Process Capability}

In this article, calculation of process capability is applicable for normal data. If in a sigma scaled graph, $99.73 \%$ observations are concentrated between $\pm 3 \sigma$ from the mean, the data distribution can be considered as normal.

Process capability indices can be divided into two groups: the indices that measure the processes potential capabilities, and the ones that measure their actual capabilities. The potential capability indices determine how capable a process is if certain conditions are met- essentially, if the mean of the process' natural variability is centered to the target of the standard specifications. The actual capability indices do not require the process to be centered to be accurate.

Potential Capability, Cp: A process is said to be capable if the spread of the natural variations fits in the spread of the specified limits. This is so when the ratio of the specified range to the control limits is greater than one. In other words, the following ratio should be greater than 1:

$$
C_{p}=\frac{U S L-L S L}{U C L-L C L}=\frac{U S L-L S L}{6 \sigma}
$$

The value of $\mathrm{Cp}=1$ if the standard specified limit equals the limit of the natural variations of the process (control limit), in which case the process is said to be barely capable; it has the 
potential to only produce non defective products if the process mean is centered to the specified target. Approximately 0.27 percent, or 2700 parts per million, are defective.

The value of $\mathrm{Cp}>1$ if the standard specified limit is greater than control limit, in which case the process is potentially capable-if the process mean is centered to the specified targetand is (probably) producing products that meet or exceed the customers' requirements.

The value of $\mathrm{Cp}<1$ if the standard specified limit is smaller than control limit. The process is said to be incapable.

Actual Process Capability, Cpk: The reason why $\mathrm{Cp}>1$ does not necessarily mean that the process is not producing defects, is that, the range of the control limits might be smaller than the one of the standard specified limits, but if the process mean is not centered to the specified target, one side of the control limit might exceed the specified limits.

If the process mean is not centered to the specified target, $\mathrm{Cp}$ would not be very informative because it would only tell which of the two ranges (process control limits and standard specified limits) is wider, but it would not be able to inform on whether the process is generating defects or not. In that case, another capability index is used to determine a process' ability to respond to customers' requirements and it is Cpk.

The Cpk measures how much of the production process really conforms to the standard specifications. The $\mathrm{k}$ in Cpk is called the k-factor; it measures the level of deviation of the process mean from the specified target.

$$
\begin{aligned}
& C_{p k}=(1-k) C_{p} \\
& k=\frac{|(U S L+L S L) / 2-\overline{\bar{X}}|}{(U S L-L S L) / 2}
\end{aligned}
$$

\section{Process Capability Analysis Assumptions}

Process capability analysis assumes whether the production process is in-control and stable or not. Because what are being compared are the specified limits and the control limits. If the process is out of control for a time period, some of the measurements might be outside the control limits and would not be taken into account. As example, isolated out of specification (OOS) should not be considered in process capability analysis. Because, that isolated OOS might be due to specific reason which can not be considered as general phenomena.

The stability of the process refers to the ability of the process auditor to predict the process trends based on past experience. A process is said to be stable if all the variables used to measure the process' performance have a consistent mean (within a specific range) and a consistent variance (within a specific range) over a sufficiently long period of time.

If the value of $\mathrm{Cpk}=1$, the process can be considered barely capable. In this case, there is a risk to fall down capability below 1 , hence process may produce defective product. 
If the value of Cpk>1, the process is capable. It means process is capable to meet or exceed customer's expectations.

If the value of $\mathrm{Cpk}<1$, the process is incapable. In this case, improvement of process or revision of specification is required.

\section{Conclusion}

Generally, processes are operated considering standard specified limit of CTQ characteristics in pharmaceutical company. It has a risk to produce defective product if process trend is shifted to any one side of specified limit or spread of natural variation (3 sigma limit) is wider than specified limit. An in-house limit can be set to mitigate the risk where in-house limit should be within standard specified limit. If processes are operated within in-house limit, 3 sigma limits for a data set will be within standard specified limits. Hence process capability will be greater than one which will indicate process is capable to meet or exceed customer's requirement.

\section{Reference}

Bass, I. Six Sigma Statistics with Excel and Minitab. USA: McGraw-Hill; 2007: 171-202

NIST/SEMATECH, e-Handbook of Statistical Methods

http://www.itl.nist.gov/div898/handbook/, date: 02 March 2013. 